\title{
Early re-brightening of the afterglow of GRB 050525a ${ }^{\star}$
}

\author{
A. Klotz ${ }^{1,2}$, M. Boër ${ }^{2}$, J. L. Atteia ${ }^{3}$, G. Stratta ${ }^{2,3}$, R. Behrend ${ }^{4}$, F. Malacrino ${ }^{3}$, and Y. Damerdji1,2 \\ 1 CESR, Observatoire Midi-Pyrénées (CNRS-UPS), BP 4346, 31028 Toulouse Cedex 04, France \\ e-mail: klotz@cesr.fr \\ 2 Observatoire de Haute-Provence, 04870 Saint Michel l'Observatoire, France \\ 3 LAT, Observatoire Midi-Pyrénées (CNRS-UPS), 14 avenue E. Belin, 31400 Toulouse, France \\ ${ }^{4}$ Observatoire de Genève, 1290 Sauverny, Switzerland
}

Received 13 June 2005 / Accepted 11 July 2005

\begin{abstract}
We present time resolved optical data acquired by the TAROT automated observatory on the afterglow of GRB 050525a from 6 to $136 \mathrm{~min}$ after the GRB. We find evidence for a rapid re-brightening of $0.65 \mathrm{mag}$ of the afterglow at $\sim 33 \mathrm{~min}$ after the GRB. The decay slope $\alpha$ is $1.14 \pm 0.07$ in the first part and is $1.23 \pm 0.27$ after the re-brightening event. The afterglow of GRB 050525a is the third known afterglow that exhibits a re-brightening event beginning at $0.01-0.02$ day in the rest time frame.
\end{abstract}

Key words. gamma-ray: bursts

\section{Introduction}

GRB 050525a was a very bright gamma-ray burst (GRB) detected on May 25th, 2005 at 00:02:53.3 UT (hereafter $t_{\text {trig }}$ ) by the BAT instrument on the Swift spacecraft (trigger $=130088$, Band et al. 2005). The gamma-ray light curve shows that GRB 050525a is a multipeak GRB, with an emission lasting approximately $10 \mathrm{~s}$ above $50 \mathrm{keV}$. The fluence of GRB $050525 \mathrm{a}$ in the range $20-1000 \mathrm{keV}$ is $7.84 \times 10^{-5} \mathrm{erg} / \mathrm{cm}^{2}$, and its peak energy is $E_{\text {peak }}=84 \mathrm{keV}$ (Golenetskii et al. 2005). Spectroscopic observations performed $11 \mathrm{~h}$ after the GRB revealed absorption lines from the host galaxy at a redshift $z=$ 0.606 (Foley et al. 2005). At that redshift, and adopting a flat cosmology with $\Omega_{\mathrm{m}}=0.3, \Omega_{\Lambda}=0.7$, and $h_{0}=0.65$, the isotropic-equivalent energy of GRB $050525 \mathrm{a}$ in the range $1 \mathrm{keV}$ to $10 \mathrm{MeV}$ is $E_{\text {iso }}=12.6 \times 10^{52} \mathrm{erg}$. The intrinsic peak energy of the time integrated spectrum is $135 \pm 2 \mathrm{keV}$.

In this letter we report the early optical observations of the afterglow of GRB 050525a, performed with the TAROT robotic observatory. The Gamma-ray bursts Coordinates Network (GCN) notice, providing celestial coordinates to ground stations, was send at 00:08:48 (Band et al. 2005), too late to detect the hypothetical optical prompt emission. The first image of TAROT started at 00:08:52.1 UT, 5 min $59 \mathrm{~s}$ after the GRB. The afterglow was detected on all images taken until the end of the night at the TAROT observatory (02:19 UT) at the coordinates quoted by Rykoff et al. (2005):

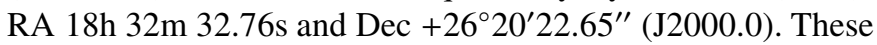

^ Based on observations performed with TAROT at the Calern observatory. data provide a continuous follow-up from 6 to 136 min after the GRB. In this paper we show that the classical exponential decay was perturbated at $t-t_{\text {trig }} \simeq 33$ min by a re-brightening event.

Section 2 describes the technical details of the TAROT observations and the data reduction. In Sect. 3, we compare our early time observations of GRB 050525a with those of other bursts with well sampled early optical observations $(t \sim$ 0.01-0.1 day). In Sect. 4 we discuss the theoretical interpretations which have been proposed to explain the early re-brightening of GRB optical afterglows.

\section{TAROT observations}

TAROT is a fully autonomous $25 \mathrm{~cm}$ aperture telescope installed at the Calern observatory (Observatoire de la Côte d'Azur, France). This telescope is devoted to very early observations of GRB optical counterparts. A technical description of TAROT can be read in Bringer et al. (1999) and in Bringer et al. (2001). The CCD camera is a commercial Andor, based on a Marconi 4240 chip, and is placed at the newtonian focus. The spatial sampling is $3.3 \mathrm{arcsec} / \mathrm{pixel}$. The field of view is $1.86^{\circ}$. The readout noise is 9 electrons rms. The readout time is $5 \mathrm{~s}$ (to read the entire $2048 \times 2048$ matrix with no binning).

The first image was taken less than $4 \mathrm{~s}$ after the position of GRB 050525a was provided by the GCN. A series of 76 images of various exposure times $(15,30,60,120 \mathrm{~s})$ were performed without any filter (hereafter clear filter). On-line preprocessing software provides calibrated images less than 2 min after they were taken (corrected by dark, flat and astrometrically calibrated from the USNO-A1.0 catalog). 
Table 1. $\log$ of the measurements. The first column is the $T$ date from GRB (in minutes) as defined by Eq. (1). The second is the $C R$ magnitude (see text) and the third is the error.

\begin{tabular}{ccc|ccc}
\hline \hline$T(\min )$ & $C R$ & $2 \sigma$ & $T(\min )$ & $C R$ & $2 \sigma$ \\
\hline 6.104 & 14.54 & 0.11 & 18.931 & 15.81 & 0.20 \\
6.471 & 14.31 & 0.09 & 20.249 & 15.97 & 0.22 \\
6.839 & 14.54 & 0.11 & 21.351 & 15.94 & 0.21 \\
7.326 & 14.66 & 0.12 & 22.452 & 16.25 & 0.31 \\
7.931 & 14.65 & 0.12 & 23.554 & 15.78 & 0.20 \\
8.536 & 14.79 & 0.21 & 25.779 & 16.19 & 0.23 \\
9.978 & 14.95 & 0.14 & 27.111 & 15.92 & 0.21 \\
10.338 & 15.08 & 0.15 & 29.351 & 16.48 & 0.25 \\
10.698 & 15.44 & 0.18 & 30.453 & 16.39 & 0.26 \\
11.172 & 15.36 & 0.17 & 31.569 & 16.64 & 0.27 \\
11.791 & 15.16 & 0.15 & 34.477 & 15.97 & 0.22 \\
12.403 & 15.25 & 0.16 & 36.580 & 15.79 & 0.26 \\
13.363 & 15.29 & 0.16 & 38.697 & 16.24 & 0.35 \\
14.472 & 15.29 & 0.16 & 42.842 & 15.88 & 0.21 \\
15.589 & 15.39 & 0.25 & 52.338 & 16.43 & 0.25 \\
16.705 & 16.12 & 0.23 & 65.275 & 16.53 & 0.26 \\
17.814 & 15.80 & 0.20 & 78.185 & 17.11 & 0.30 \\
18.931 & 15.81 & 0.20 & 108.318 & 17.36 & 0.32 \\
\hline
\end{tabular}

On the first 31 images, the afterglow is bright enough to be measured with a good accuracy on individual images. Later images were co-added to increase the signal to noise ratio. Due to the decreasing flux during exposures, the mean date of an observation, $T$, is not the middle of exposure; it must be interpolated between $t_{1}$ (start of the first exposure) and $t_{2}$ (end of the last exposure) such that the flux $f$ verifies:

$\int_{t_{1}}^{t_{2}} f(t) \mathrm{d} t=f(T) \int_{t_{1}}^{t_{2}} \mathrm{~d} t$.

Considering afterglow decay flux law: $f(t) \propto t^{-\alpha}$, and assuming all times counted since $t_{\text {trig }}$,

$T=\frac{t_{2}-t_{1}}{\ln \left(t_{2} / t_{1}\right)} \quad$ if $\alpha=+1$

$T=\left[\frac{\left(t_{2}-t_{1}\right) \cdot(1-\alpha)}{t_{2}{ }^{1-\alpha}-t_{1}{ }^{1-\alpha}}\right]^{1 / \alpha} \quad$ if $\alpha \neq+1$

$\alpha$ and $T$ values are computed by iterations. Initial $T$ values are computed taking $\alpha=+1$. Then the fit of the first light-curve refines the $\alpha$ value. The second iteration is enough to converge (see Table 1 and Fig. 1).

The presence of the star USNO-B1 1163-0325216 $(R \sim$ 16.6), at about 15 arcsec north-west from the afterglow, perturbated the photometry, especially at the end of the night. The set of magnitudes provided by Klotz et al. (2005) was affected by this effect, which led to a false plateau 40 min after the trigger. To eliminate this effect, we computed an image (designated hereafter mask) of the field which does not show the afterglow. The mask is synthetized in two steps: first we oversampled the 76 images by a factor three and stacked them to synthetize the
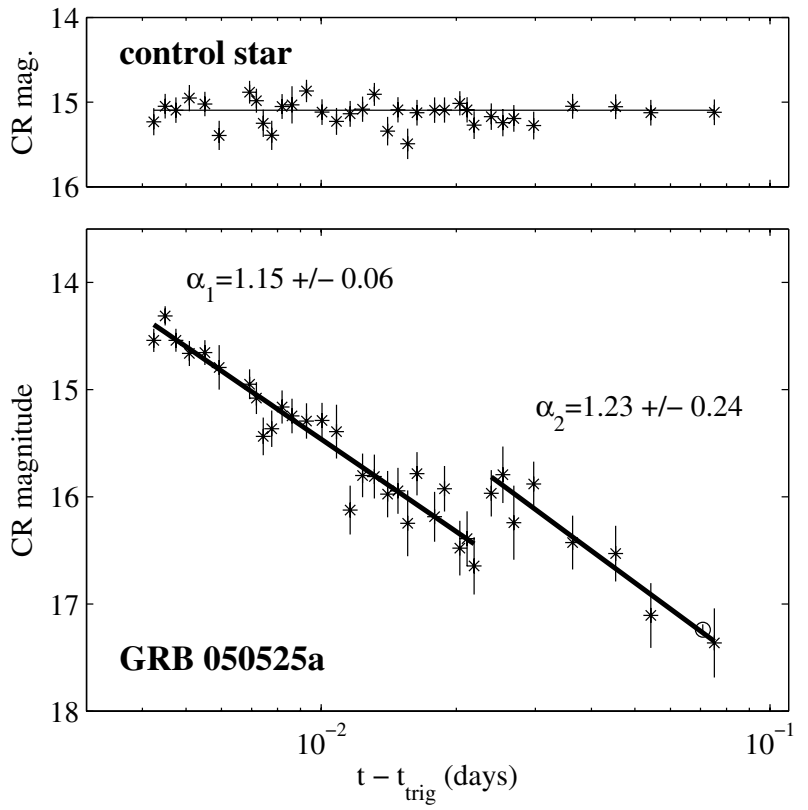

Fig. 1. Top: photometry of the control star USNO-B1 1163-0325198. Bottom: GRB 050525a afterglow light-curve obtained by TAROT (data from Table 1). Straight lines are linear fits in the logarithm time scale and their corresponding slope values. Error bars are $2 \sigma$ uncertainties (95\% of confidence). The symbol $\circ$ is a $R$ measurement performed by Malesani et al. (2005) with the Italian $3.6 \mathrm{~m} \mathrm{TNG}$ and calibrated with Landolt stars.

sum. In this image, the afterglow and the star are well separated. The second step is to clear only the afterglow spot in order to synthetize the mask. The mask was normalized in flux for each image and was substracted, leading to images where only the afterglow appears (and some residuals of bright stars). Then we extracted the magnitude of the afterglow avoiding problems of contamination by the nearby star.

The afterglow of GRB 030329 shows no color evolution during the first phases of decay (Zeh et al. 2003a,b). Taking advantage of this fact, we performed differential photometry with two stars as reference: USNO-B1 1163-0325130 and USNO-B1 1163-0325158. We choose USNO-B1 1163-0325198 as a star to control the stability of photometry and to verify that the magnitude of reference stars does not vary. As we have no information about the afterglow colours (we used no filter), we cannot obtain $R$ magnitudes directly. Moreover, the USNO-B1 catalog gives photographic $R$ magnitudes which cannot be used to calibrate magnitudes finely. However, as the airmass only varied from 1.13 to 1.05 during measurements, its effect on colours is assumed to be negligible (presumatly lower than 0.05 mag, much less than other uncertainties). As a consequence, our differential magnitudes can be related to the $R$ band by a simple offset. Fortunately, Malesani et al. (2005) and Malesani (2005) measured $R=17.24 \pm 0.05,0.0694$ day after the GRB (about the same time than our last measurement) using a large aperture telescope, a $R$ filter and a calibration by Landold stars. Then, we applied an offset to our differential magnitudes to be compatible with the Malesani measurement. We designate as $C R$, our unfiltered magnitudes calibrated in the $R$ band. 
The light-curve (Fig. 1) shows two distinct parts separated at $t-t_{\text {trig }} \simeq 33 \mathrm{~min}$. Fits of the decay slopes give $\alpha_{1}=1.14 \pm$ 0.07 and $\alpha_{2}=1.23 \pm 0.27$. Uncertainties are such that these slopes are not significantly different. We can join the two curves by two extreme paths: i) a sharp re-brightening of $0.65 \mathrm{mag}$ in less than 3 min centered at $t-t_{\text {trig }}=32.8 \mathrm{~min}$, ii) a plateau (flat re-brightening) of $C R \simeq 16.1$, beginning at $t-t_{\text {trig }} \simeq 26 \mathrm{~min}$ and finishing at $t-t_{\text {trig }} \simeq 43 \mathrm{~min}$. The magnitude uncertainties of our measurements are too large to discriminate between these two assumptions.

To summarize, the re-brightening of the afterglow of GRB 050525a is real, the duration of the transition is comprised between less than $3 \mathrm{~min}(\delta t / t=0.1$ and a factor two in brightness) and up to $17 \mathrm{~min}(\delta t / t=0.5$ and a plateau) centered at $t-t_{\text {trig }}=32.8 \mathrm{~min}$, and the slopes of the temporal decay before and after the re-brightening are fully comparable.

\section{Early optical GRB afterglows}

Few GRB afterglows have been observed at optical wavelengths less than one hour after the GRB. They include GRB $990123(z=1.60)$, GRB $021004(z=2.33)$, GRB 021211 $(z=1.01)$, GRB 030418, GRB 041219a, GRB $050319(z=$ 3.24), GRB 050502a $(z=3.79)$, and GRB 050525a $(z=0.606)$ discussed in this paper. Within this small sample, short-scale variability seems to be the rule rather than the exception. This variability can be observed as single or multiple re-brightenings (GRB 021004, GRB 041219a, GRB 050319, GRB 050525a), as a shallowing (GRB 990123, GRB 021211) or a steepening (GRB 050319) of the light-curve, or as a gradual rise of the afterglow (GRB 030418).

Figure 2 compares the early optical afterglows of four GRBs with a measured redshift (GRB 050502a is not included in this comparison since the data on its early afterglow have not yet been published). For a proper comparison, the abscissa of the plot gives the time after the trigger, in the referential of the GRB. A striking feature is the presence of an episode of re-brightening starting 0.01 to 0.02 days after the trigger, in three out of the four GRBs displayed in Fig. $2^{1}$. At first glance the three GRBs which exhibit re-brightnening episodes do not have special properties. GRB 040319 is a single pulse GRB, while GRB 021004 and GRB 050525a are multi-peak events. The isotropic-equivalent energies and rest frame peak energies of GRBs in Fig. 2 are $12.6 \times 10^{52}$ erg and $135 \mathrm{keV}$ for GRB $050525 \mathrm{a}, \sim 3.1 \times 10^{52} \mathrm{erg}$ for GRB $050319^{2}, 5.1 \times$ $10^{52} \mathrm{erg}$ and $266 \mathrm{keV}$ for GRB 021004 , and $1.4 \times 10^{52} \mathrm{erg}$, and $92 \mathrm{keV}$ for GRB 021211. Since three out of four GRBs with early optical follow-up exhibit re-brightening episodes, it is tempting to conclude that this is a common feature of GRB afterglows. This remark clearly points out the necessity of very quick optical follow-up to measure the decay slope before 0.01 day (14 $\mathrm{min}$ ), required to assess the time and amplitude of possible re-brightening episodes in future GRBs.

${ }^{1}$ GRB 041219a, whose redshift is not known, exhibits a rebrightening episode starting $\sim 17 \mathrm{~min}$, or 0.012 day, after the trigger (Blake et al. 2005).

2 The peak energy of GRB 050319 is not known.

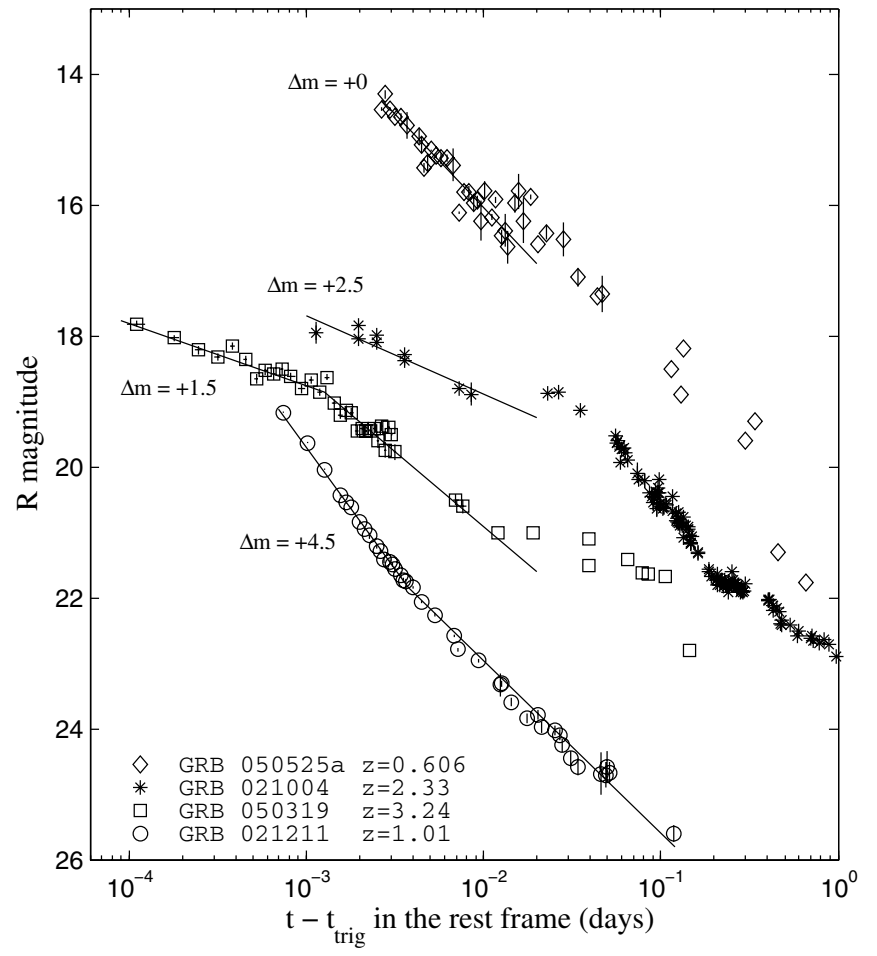

Fig. 2. Optical light-curves of some early afterglows scaled in their rest time frame. Curves are vertically shifted by $\Delta m$ values to avoid crossing of curves. The upper three curves show re-brightening events at $\sim 1-2 \times 10^{-2}$ day. Data for GRB 050525a are from TAROT, and GCNCs 3465, 3469, 3470, 3489, 3491, 3488, 3493, 3486, 3506. Data for GRB 021004 are from Fox et al. (2003a) and GCNCs 1564, $1566,1570,1573,1576,1577,1578,1580,1581,1582,1584,1587$, $1591,1594,1606,1614,1615,1628$. Data for GRB 050319 are from Woźniak et al. (2005) and GCNCs 3120, 3121, 3124, 3130, 3139. Data for GRB 021211 are from Holland et al. (2002), Li et al. (2003), Fox et al. (2003b).

\section{Discussion and conclusions}

Within the context of the internal/external shock model of GRBs, re-brightening episodes have been explained by the reverse shock, by a continuing activity of the central engine, by a variable density profile of the external environment in which the fireball expands, by the presence of neutrons in the ejecta, or by the destruction of dust surrounding the source. Late afterglow emission is explained as the forward shock component of external shock produced by the interaction of the expanding fireball with the external medium.

Variable energy input model predicts early light-curve rebrightenings (Nakar et al. 2003). The energy variability could be provided by refreshed shocks produced by slow, massive shells ejected late in the GRB, that collide with the inital blast wave when it has decelerated. After each collision, the flux from the fireball increases but the light-curve decay will continue with the same rate as prior to the collision. The net result is a shift upward of the initial light-curve at the time of the collision with same decay rate as before collision (Bjornsson et al. 2004). Alternatively, a variable energy input coud be due to initial energy (per solid angle) inhomogeneities in the jet, like in the patchy shell model described by Kumar \& Piran (2000b). 
In the variable density scenario, clumpy inter-stellar matter (ISM) or variable wind expelled from the massive progenitor, produces high density regions that, interacting with the expanding fireball, could provide flux enhancements if particular electron cooling conditions are satisfied (e.g. Lazzati et al. 2002). In this case, in fact, the flux would be sensitive to density variations only in the "slow cooling regime", with the observed frequency below the cooling frequency (Sari et al. 1998). The recovery of the initial slope after the bump could be achieved requiring a decrease in the density below the initial value immediately after the high density region (Lazzati et al. 2002).

Afterglow re-brightening has been predicted also by the neutron-fed afterglow model when the already decelerating ion shell sweeps up the trail of decay products left from the ahead decaying neutron shell. The arrival time of the re-brightening depends on the Lorentz factor of the neutron shell and can vary from few seconds to several ten days after the burst (Beloborodov 2003).

Another explanation is that dust destruction mechanisms might provide an enhancement of the observed optical flux within a time scale that depends, among several other parameters, on the density distribution of the circumburst dust. A decreasing of reddening is predicted in this case (Perna et al. 2003).

Finally, very early optical emission is thought to be an effect of the reverse shock component of the external shock. The emergence of the forward shock from the reverse shock emission component produces a shallowing of the early-time light-curve after an initial steep decay that can mimick a rebrightening (Panaitescu \& Kumar 2004). Indeed this model has been recently invoked to explain the phenomenology of GRB 050525a (Shao \& Dai 2005). Our data are consistent with this model, althought at this stage we do not know if they favour this model with respect to the others.

Detailed observations of the early afterglow are essential to unravel the physical processes acting within the ejecta and when the ejecta starts to interact with the medium surrounding the source. Critical observations include the timescale of luminosity variations, the correlation of luminosity variations with changes of optical colors, and the polarization of the optical emission. Another important clue will be provided by the monitoring of the shape of the multi-wavelength spectrum (from optical to X-rays) of the early afterglow. GRB 050525a is, after GRB 050319, the second GRB with a known distance, with detailed observations of the early afterglow at optical (this paper) and X-ray wavelengths (Band et al. 2005). This proves that, eigth years after the discovery of GRB afterglows, a new window opens: multi-wavelength observations of the very early afterglow. This remarkable advance has been possible thanks to the availability of arcminute localizations quickly distributed to efficient robotic telescopes, and to the excellent performance of the XRT on-board SWIFT. The observations presented in this paper confirm the richness of the information contained in the very early afterglow, and the great promises of the multiwavelength observations which are now within our range.

Acknowledgements. The TAROT telescope has been funded by the Centre National de la Recherche Scientifique (CNRS), Institut National des Sciences de l'Univers (INSU) and the Carlsberg Fundation. It has been built with the support of the Division Technique of INSU. We thank the technical staff contributing to the TAROT project: G. Bucholtz, J. Esseric, C. Pollas and Y. Richaud. We thank Dr. Malesani who kindly provide us the refined value of its observation quoted in the GCNC 3469 and for helpful discussions about photometry.

\section{References}

Band, D., Cummings, J., Perri, M., et al. 2005, GCNC, 3466 Beloborodov, A. M. 2003, ApJ, 585, L19

Bjornsson, G., Gudmundusson, E. H., \& Johannesson, G. 2004, ApJ, 615, L77

Blake, C. H., Bloom, J. S., Starr, D. L., et al. 2005, Nature, 435, 181

Bringer, M., Boër, M., Peignot, C., et al. 1999, A\&AS, 138, 581

Bringer, M., Boër, M., Peignot, C., et al. 2001, Exper. Astrophys., 12, 34

Foley, R. J., Chen, H.-W., Bloom, J., et al. 2005, GCNC, 3483

Fox, D. W., Yost, S., Kulkarni, S. R., et al. 2003a, Nature, 422, 284

Fox, D. W., Price, P. A., Soderberg, A. M., et al. 2003b, ApJ, 586, L5

Golenetskii, S., Aptekar, R., Mazets, E., et al. 2005, GCNC, 3474

Holland, S., Bersier, D., Bloom, J. S., et al. 2004, AJ, 128, 1955

Klotz, A., Boër, M., \& Atteia, J. L. 2005, GCNC, 3473

Kumar, P., \& Piran, T. 2000b, ApJ, 532, 286

Lazzati, D., Rossi, E., Covino, S., et al. 2002, A\&A, 396, L5

Li, W., Filippenko, A. V., Chornock, R., \& Jha, S. 2003, ApJ, 586, L9

Malesani, D. 2005, private communication

Malesani, D., Piranomonte, S., Fiore, F., et al. 2005, GCNC, 3469

Nakar, E., Piran, T., \& Granot, J. 2003, New Astron., 8, 495

Panaitescu, A., \& Kumar, P. 2004, MNRAS, 353, 511

Perna, R., Lazzati, D., \& Fiore, F. 2003, ApJ, 585, 775

Rykoff, E. S., Yost, S. A., \& Swan, H. 2005, GCNC, 3465

Sari, R., Piran, T., \& Narayan, R. 1998, ApJ, 497, L17

Shao, L., \& Dai, Z. G. 2005, ApJ, submitted

[arXiv: astro-ph/0506139]

Woźniak, P. R., Vestrand, W. T., Wren, J. A., et al. 2005, ApJ, 627, L13

Zeh, A., Klose, S., Nysewander, M., Reichart, D., \& Greiner, J. 2003a, GCNC, 2047

Zeh, A., Klose, S., Henden, A., \& Greiner, J. 2003b, GCNC, 2115 